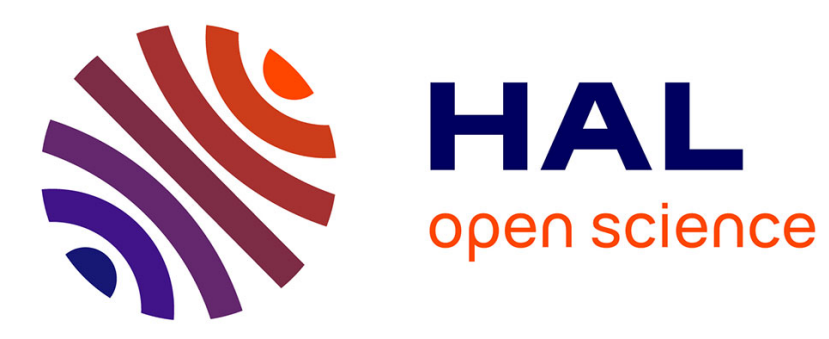

\title{
A unique formalism for VLSI logical and electrical synthesis
}

J. Frehel, J.-C. Longchambon, P. Malardier

\section{To cite this version:}

J. Frehel, J.-C. Longchambon, P. Malardier. A unique formalism for VLSI logical and electrical synthesis. Revue de Physique Appliquée, 1987, 22 (1), pp.21-28. 10.1051/rphysap:0198700220102100 . jpa-00245510

\section{HAL Id: jpa-00245510 https://hal.science/jpa-00245510}

Submitted on 1 Jan 1987

HAL is a multi-disciplinary open access archive for the deposit and dissemination of scientific research documents, whether they are published or not. The documents may come from teaching and research institutions in France or abroad, or from public or private research centers.
L'archive ouverte pluridisciplinaire HAL, est destinée au dépôt et à la diffusion de documents scientifiques de niveau recherche, publiés ou non, émanant des établissements d'enseignement et de recherche français ou étrangers, des laboratoires publics ou privés. 


\title{
A unique formalism for VLSI logical and electrical synthesis
}

\author{
J. Frehel, J.-C. Longchambon and P. Malardier \\ Thomson CSF Service CSAO, B.P. 54, 38120 St-Egrève, France \\ (Reçu le 19 juillet 1986, accepté le 16 octobre 1986)
}

\begin{abstract}
Résumé. - Ce papier présente un formalisme unique des représentations des connaissances logiques et électriques d'un concepteur de circuits digitaux MOS en terme de règles de réécriture. Ce formalisme permet de résoudre des problèmes de synthèse logique depuis une description fonctionnelle vers un réseau de portes logiques, de synthèse électrique depuis une description en termes de portes vers un réseau de transistors MOS, d'extraction logique depuis un réseau de transistors vers une description booléenne. Les moteurs d'inférence correspondant à ces différents outils utilisent tous un mécanisme d'unification commutative à arité variable.
\end{abstract}

\begin{abstract}
This paper presents a unique formalism of knowledge representation of a MOS digital designer in terms of sets of rewriting rules. This formalism provides solution to logical synthesis from functional description to gate network, to electrical synthesis from gate network to equivalent MOS transistor network and to logical extraction from a transistor network. Corresponding inference origines extensively use a general commutative unification process with variable arity of operators.
\end{abstract}

\section{Introduction.}

This research has been developed in the framework of the Sycomore VLSI French National project. The main objective was to develop a unique formalism that could support symbolic manipulation, for logical and electrical synthesis as well as logical and functional extraction.

Three levels of description will be discussed in this paper. Logical level is the world of logical gates and Boolean expressions. In a switch level description basic devices are switches assembled in series and parallels. Both logical and switch level are unconcerned with technological considerations while the electrical level is strongly connected with MOS technology since it explicitly manipulates $\mathrm{P}$ and $\mathrm{N}$ transistors.

Synthesis tools and procedures are viewed as automatic translators from an upper description level to a lower one. They must guarantee that functionality is preserved all along this downward synthesis process.

Extraction tools are able to extract functionality from switch level or electrical level. All of them are based on symbolic calculus and rule based expert systems.

Since many equivalent electrical implementations are available for a given functionality there is not a unique synthesis tool but a lot of them, the choice between them has to be made interactively by the designer trying to solve the classical tradeoffs of speed area and power. The system can be viewed as an extensible designer assistant the knowledge of which is imbedded in sets of rewriting rules. As for any other symbolic calculus system rule application is done through a general unification process. Since most of the operators which are beeing used are commutative and of unknown arity a general commutative unification algorithm has been developed. This implies that a backtracking mechanism is included in the unification algorithm.

Furthermore, for sake of efficiency, unification is only attempted on a filtered sub-set of rules.

Most of the algorithms which are presented in the paper will not be described in full detail but will be only outlined. Some of them have needed a great effort of optimization in terms of complexity and efficiency. Most of the inference engines which have been developed in the system use heuristics which shall not be discussed here. The system also includes a symbolic layout system and a crystal like timing analyser which will be described elsewhere.

I finally would thank all the people of our team in Thomson csf who have participated to this research and have implemented these ideas in a CAD system.

\section{Elementary operators.}

All operators are used in a prefixed notation. Logic and circuitry are systematically described expressing the outputs in terms of the inputs and using formulae 
bidirectional signals will be the subject of a separate section.

2.1 LOGICAL OPERATORS. - They are the classical ones of Boolean algebra : Or And Not Nor Nand. One output $\mathrm{s}$ is described by a Boolean expression of the type :

$s=(\operatorname{Or}($ And a p $(\operatorname{Not} c))($ And e $(\operatorname{Not} f)))$

where $a b c d e f$ are either input or internal signals. Variables which represent signals can be indexed so for example that the output cary of the bit $i$ of an adder can be described by the recursive formula :

$$
\begin{aligned}
\mathrm{c}[i]=(\text { Or } & (\text { And } \mathrm{a}[i] \mathrm{b}[i](\operatorname{Not} \mathrm{c}[i-1])) \\
& (\text { And }(\text { Not } \mathrm{a}[i]) \mathrm{b}[i] \mathrm{c}[i-1]) \\
& (\text { And } \mathrm{a}[\mathrm{i}](\operatorname{Not} \mathrm{b}[i]) \mathrm{c}[i-1]) \\
& (\text { And } \mathrm{a}[i] \mathrm{b}[i] \mathrm{c}[i]))
\end{aligned}
$$

This formula has to be immediately completed by the one which gives carry for $i=0$.

$$
\mathrm{c}[i](i=0)=\mathrm{c} 0 \text {. }
$$

The condition $(i=0)$ is a predicate that restricts the application of the formula (see later the chapter on predicates). For seak of simplicity (Not a) will be also noted /a.
2.2 OPERATORS ON SWITCHES. - They are $P$ to put switches in parallel

$\mathrm{S}$ to out switches in series

$\mathrm{Nd}$ to connect admittances to sources of power.

The following network of switches:

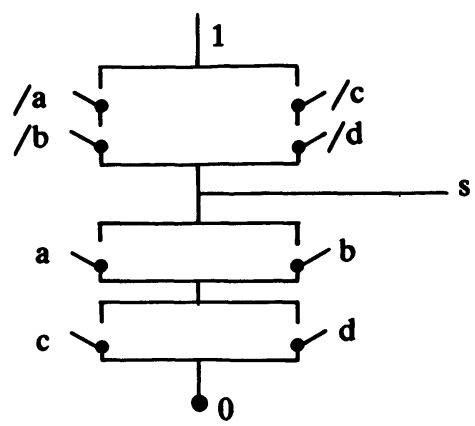

will be described by the formula :

$$
\begin{aligned}
& \mathrm{S}=(\mathrm{Nd}(\mathrm{P}(\mathrm{S} / \mathrm{a} / \mathrm{b})(\mathrm{S} / \mathrm{c} / \mathrm{d})) \leftarrow 1 \\
&(\mathrm{~S}(\mathrm{P} \mathrm{a} \mathrm{b})(\mathrm{P} \mathrm{c} \mathrm{d})) \leftarrow 0)
\end{aligned}
$$

2.3 CMOS TRANSISTORS REPRESENTATION. - $\mathrm{N}$ channel and $\mathrm{P}$ channel transistors of CMOS technology are described by two operators Trn and Trp.

A CMOS implementation of the brevious switch network will be :

$$
\begin{array}{r}
\mathrm{S}=(\operatorname{Nd}(P(S(\operatorname{Trp} a)(\operatorname{Trp} b))(S(\operatorname{Trp} c)(\operatorname{Trp} d))) \leftarrow 1 \\
(S(P(\operatorname{Trn} a)(\operatorname{Trn} b))(P(\operatorname{Trn} c)(\operatorname{Trn} d))) \leftarrow 0)
\end{array}
$$

in which 1 and 0 represent respectively power and ground. The corresponding schematic is :

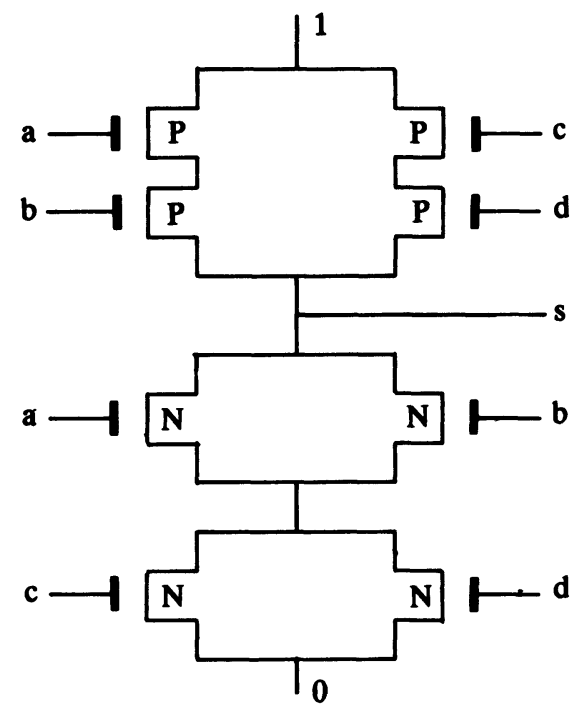

2.4 BIDIRECTIONAL ADMITTANCES AND SIGNALS. - Very few pieces of circuitry use in fact pass- transistor bidirectional property ; most of the time they are memory devices or clocked transmission gates connected to busses. A flat fully expanded description generally leads to an artificial multiplication of these bidirectional devices although they do not work in a true bidirectional mode.

A bidirectional admittance is as any other one composed of series and parallels of transistors ; the difference is it is not connected to power or ground but to an other type of source which is itself a node of other admittances. Such a bidirectional admittance has two extreme signals that we have to name so that we can express each one in terms of the other when the admittance is closed.

We shall have to write (explicitely or implicitely) this equality; for example the following electrical schematic :

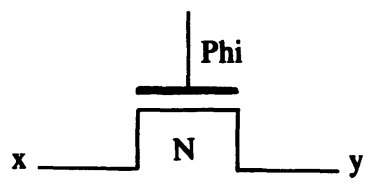


will be described by the following equations :

$$
\begin{aligned}
\mathbf{y} & =(\operatorname{Nd}(\operatorname{Trn} \text { phi }) \rightarrow \mathrm{x}) \\
\mathbf{x} & =(\operatorname{Nd}(\operatorname{Trn} \text { phi }) \rightarrow \mathrm{y})
\end{aligned}
$$

The classical memory point will then be written :

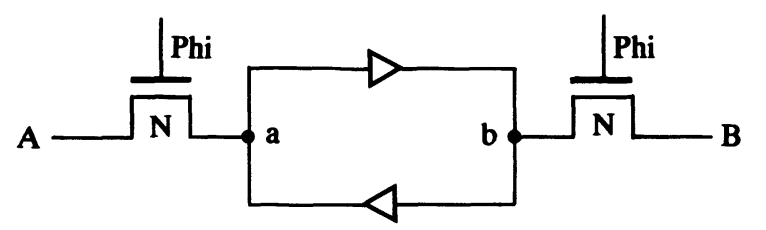

$$
\begin{aligned}
& \mathbf{A}=(\mathbf{N d}(\operatorname{Trn} \text { phi }) \rightarrow \mathrm{a}) \\
& \mathrm{a}=(\mathbf{N d}(\operatorname{Trn} \text { phi }) \rightarrow \mathbf{A}) \\
& \mathbf{B}=(\mathbf{N d}(\operatorname{Trn} \text { phi }) \rightarrow \mathrm{b}) \\
& \mathbf{b}=(\mathbf{N d}(\operatorname{Trn} \text { phi }) \rightarrow \mathrm{B}) \\
& \mathrm{a}=/ \mathrm{b} \\
& \mathbf{b}=/ \mathrm{a}
\end{aligned}
$$

2.5 Indices AND PREDiCATes. - Predicates on indices will be used to specify the domain of indices on which a formula is valid. These predicates are constructed with the classical arithmetic and logic operators $t^{*}-1><>=<=$ And Or Not. The following two formulae

$$
\begin{aligned}
& \mathrm{s}[i]=(\text { And } \mathrm{a}[i] \mathrm{s}[i-1]) \\
& \mathrm{s}[i](i=0)=\mathrm{s} 0
\end{aligned}
$$

mean that first one is valid for any value of $i$ except for $i=0$.

2.6 EVAL OPERATOR. - The Eval operator also noted, (comma) allows to specify to the system that the following expression has to be evaluated. Using formulae (4)

, $\mathrm{s}[i](i=2)$ will evaluate to : (And a [2] $\mathrm{s}[1]$ )

Using different formulae (5) :

$$
\begin{aligned}
& \mathrm{s}[i]=(\text { And } \mathrm{a}[i], \mathrm{s}[i-1]) \\
& \mathrm{s}[i](i=0)=\mathrm{s} 0
\end{aligned}
$$

we shall obtain for $s[4]$

$$
(\text { And a [4] (And a [3] (And a [2] (And a [1] s0) ) ) ) }
$$

The operator Eval therefore enables to monitor in detail recursions and evaluations.

2.7 BLOC ASSEMBLY, INTERCONNEXIONS OF BLOCS. - A hardware description language has to be hierarchical and describe connexions between blocs. The following connexion between two blocs $\mathrm{A}$ and $\mathrm{B}$ with respective outputs and inputs $(s t)(a b)$ is then expressed by the formulae :

$$
\begin{aligned}
& \{\text { B }\}: \mathrm{a}=\{\mathrm{A}\}: \mathrm{s} \\
& \{\mathrm{B}\}: \mathrm{b}=\{\mathrm{A}\}: \mathrm{t}
\end{aligned}
$$

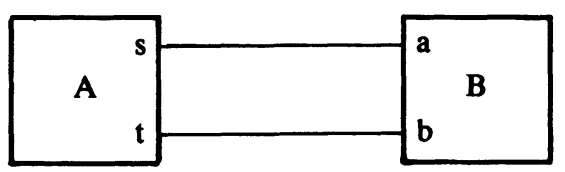

The reader will note that the corresponding interconnexion signals are not named in such a description. Of course such interconnexion equations can be indexed which makes these interconnexion equations easy to write and powerfull.

\section{Synthesis tools.}

3.1 Logical SYNTHESIS. - Their objective is to generate logical descriptions. Some of them are acquisition tools others are symbolic manipulation such as change of variables and Boolean reduction.
3.1.1 Acquisition of a formula through a truth table. Let us examine again the output carry of bit $i$ of the adder. Its truth table is :

$\begin{array}{ccccc}\begin{array}{c}\text { Line } \\ \text { number }\end{array} & \mathrm{a}[i] & \mathrm{b}[i] & \mathrm{c}[i-1] & \mathrm{c}[i] \\ \overline{0} & \overline{0} & \overline{0} & - & - \\ 1 & 0 & 0 & 0 & 0 \\ 2 & 0 & 0 & 1 & 0 \\ 3 & 0 & 1 & 0 & 0 \\ 4 & 1 & 1 & 1 & 1 \\ 5 & 1 & 0 & 0 & 0 \\ 6 & 1 & 1 & 1 & 1 \\ 7 & 1 & 1 & 1 & 1\end{array}$

The value of $c[i]$ is fully characterized by the list of the line numbers for which its value is 1 . We shall use the tool Bool1 with the following syntax :

$$
\mathrm{c}[i]=(\text { Bool1 In } \mathrm{a}[i] \mathrm{b}[i] \mathrm{c}[i-1] \text { Val } 3567)
$$

The system will rebuilt the previous formula through a well known algorithm. If the user had wanted to describe $\mathrm{c}[i]$ through the lines in which its value is 0 he would have used the tool Bool0 as follows :

$$
\mathrm{c}[i]=(\text { Bool0 In } \mathrm{a}[i] \mathrm{b}[i] \mathrm{c}[i-1] \text { Val } 0124)
$$

3.1.2 The use of recursion. - It enables the user to generate complex Boolean formulae using evaluation; 
for example one can make the carry formula recursive as follows :

$$
\begin{aligned}
\mathrm{c}[i]=(\operatorname{Or} & (\text { And } \mathrm{a}[i] \mathrm{b}[i], \mathrm{c}[i-1]) \\
& (\text { And } / \mathrm{a}[i] \mathrm{b}[i], \mathrm{c}[i-1]) \\
& (\text { And } \mathrm{a}[i] / \mathrm{b}[i], \mathrm{c}[i-1]) \\
& (\text { And } \mathrm{a}[i] \mathrm{b}[i], / \mathrm{c}[i-1])) \\
& \mathrm{c}[i](i=0)=\mathrm{c} 0
\end{aligned}
$$

so that the evaluation of , $\mathrm{c}[i](i=4)$ gives the explicit expression of $c[i]$ in terms of $a[0] b[0] a[1]$ $\mathrm{b}[1] \ldots \mathrm{a}[4] \mathrm{b}[4]$.

3.1.3 Boolean reduction. - This is a symbolic calculus tool using a large set of rewriting rules expressing distributivity and absorption properties of Boolean algebra to compute a normal disjunctive form of any Boolean expression. Temptation is great to make this tool a standard operator so that one can write :

$$
\begin{aligned}
c[i]=(\operatorname{Red}(\operatorname{Or} & (\text { And } \mathrm{a}[i] \mathrm{b}[i] \mathrm{c}[i-1]) \\
& (\text { And } / \mathrm{a}[i] \mathrm{b}[i] \mathrm{c}[i-1]) \\
& (\text { And } \mathrm{a}[i] / \mathrm{b}[i] \mathrm{c}[i-1]) \\
& (\text { And } \mathrm{a}[i] \mathrm{b}[i] / \mathrm{c}[i-1])))
\end{aligned}
$$

so that evaluation of $c[i]$ will give :

$$
\begin{aligned}
\mathrm{c}[i]=(\text { Or } & (\text { And } \mathrm{a}[i] \mathrm{b}[i]) \\
& (\text { And } \mathrm{b}[i] \mathrm{c}[i-1]) \\
& (\text { And } \mathrm{a}[i] \mathrm{c}[i-1]))
\end{aligned}
$$

A great effort has been done to optimize this operator so that it can be run very quickly even when the potential number of minterms is tremendous (millions or more). For that purpose special distributivity rules including absorption have been used. Furthermore before reduction attempt is performed on one expression the recursive process by which it has been built is analysed by the system. This allows that any subexpression which is repeatedly used in a bigger one is only reduced once. The main idea is that any big Boolean expression has necessarly been built by a program using some recursive mechanism.

3.2 Symbolic manipulation tOOLS. - Symbolic manipulation tools are numerous in the system (approximatively 30 ) therefore we shall only discuss some of them.

3.2.1 Simple variable change. - Let us examine one formula $s=($ Or (And $a b) c d)$; let us assume also $y=($ And $a b)$. The problem we want to solve is to express $\mathrm{s}$ in terms of $\mathrm{y}$ and $/ \mathrm{y}$ as much as possible. For $\mathrm{s}$ we get $\mathbf{s}=$ (Or $y c d)$.

This variable change is performed using systematically the following rewriting rules :

$$
\begin{aligned}
& (\text { And } a b) \rightarrow y \\
& (\text { Or } / a / b) \rightarrow / y
\end{aligned}
$$

3.2.2 A more complex variable change problem. Rewrite one expression $\mathrm{s}$ in terms of two given Boolean expressions $\mathrm{y}$ and $\mathrm{z}$. Let us start again with carry formula (9).

$$
\begin{aligned}
\text { Let } \mathrm{y}[i] & =(\text { Or } \mathrm{a}[i] \mathrm{b}[i]) \\
\text { and } \mathrm{z}[i] & =(\text { And } \mathrm{a}[i] \mathrm{b}[i])
\end{aligned}
$$

we want to automatically find :

$$
\mathrm{c}[i]=(\text { Or } \mathrm{z}[i](\text { And } \mathrm{c}[i-1] \mathrm{y}[i]))
$$

which can be achieved through the use of the following rewriting rules :

$$
\begin{aligned}
& (\mathrm{Or} \mathrm{a}[i] \mathrm{b}[i]) \rightarrow \mathrm{y}[i] \\
& (\text { And } \mathrm{a}[i] \mathrm{b}[i]) \rightarrow \mathrm{z}[i] \\
& (\text { And } / \mathrm{a}[i] / \mathrm{b}[i]) \rightarrow / \mathrm{y}[i] \\
& (\text { Or } / \mathrm{a}[i] / \mathrm{b}[i]) \rightarrow / \mathrm{z}[i] \\
& (\text { Or }(\text { And } / \mathrm{a}[i] \mathrm{b}[i])(\text { And } \mathrm{a}[i] / \mathrm{b}[i])) \rightarrow(\text { And } \mathrm{y}[i] / \mathrm{z}[i]) \\
& (\text { Or }(\text { And } \mathrm{a}[i] \mathrm{b}[i])(\text { And } / \mathrm{a}[i] / \mathrm{b}[i])) \rightarrow(\text { Or } / \mathrm{y}[i] \mathrm{z}[i])
\end{aligned}
$$

These rewriting rules have to be dynamically generated with respect to the considered $\mathrm{y}$ and $\mathrm{z}$ expressions. The set of possible combinations of $\mathrm{y}$ and $\mathrm{z}$ are limited to :

$$
\begin{aligned}
& y z / y / z(\operatorname{Ory} z)(\operatorname{And} y z)(\operatorname{Ory} / z)(\operatorname{Or} / y z) \\
& (\text { And } y / z)(\operatorname{And} / y z)(\operatorname{Or} / y / z)(\operatorname{And} / y / z)
\end{aligned}
$$


In the previous exemple some of the combinations do not lead to reasonable rules. For example :

$$
\begin{aligned}
(\text { And } / \mathrm{y}[i] \mathrm{z}[i]) & =(\text { And }(\text { And } / \mathrm{a}[i] / \mathrm{b}[i]) \\
& (\text { And } \mathrm{a}[i] \mathrm{b}[i])) \\
& =0,
\end{aligned}
$$

which would generate the rule :

$$
0 \rightarrow(\text { And } / \mathrm{y}[i] \mathrm{z}[\mathrm{i}]) \text {. }
$$

Therefore the system has to filter the rules to avoid cycles.

3.3 SWITCH SYNTHESIS. - From a logical description we want to generate a swich network of « equivalent functionality ». To the logic function (And $\mathrm{a} b \mathrm{c}$ ) we shall associate the following network :

$$
(\mathrm{Nd}(\mathrm{S} \mathrm{a} \mathrm{b} \mathrm{c}) \rightarrow 1)
$$<smiles>IC(I)I</smiles>

b

$$
\text { c }
$$

The logic function (Or $a b c$ ) will be translated into :

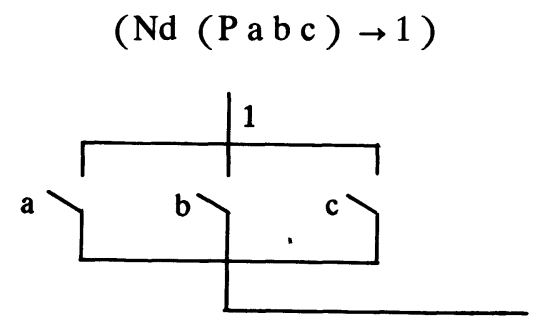

This means that the operator $\mathrm{Nd}$ brings back through the admittance $(\mathrm{P} \mathrm{a} \mathrm{b} \mathrm{c} \mathrm{)} \mathrm{the} \mathrm{logic} \mathrm{value} 1$ whenever any of the switches $a b c$ is on. We have following equivalences :

$$
\begin{aligned}
& (\text { Or } a b c)=1 \Leftrightarrow(\mathrm{P} a b c) \text { on } \\
& (\text { And } a b c)=1 \Leftrightarrow(\mathrm{S} a b c) \text { on }
\end{aligned}
$$

Corresponding rules express the isomorphism of Boolean algebra to the switch algebra with operators $\mathrm{P}$ and $\mathrm{S}$.

3.4 EleCtRiCAl SyNTHESIS. - Through the switch synthesis the logical functionality has not been altered but nothing has been said concerning the technology of electrical implementation. Electrical synthesis first needs to know the technology (NMOS true CMOS precharged CMOS domino CMOS...). Some examples of electrical implementations are as follows :

Logical level

$$
\mathrm{s}=(\mathrm{N} \text { and } \mathrm{a} \mathrm{b})
$$

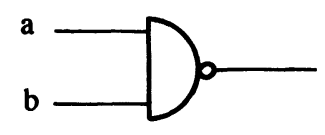

Switch level

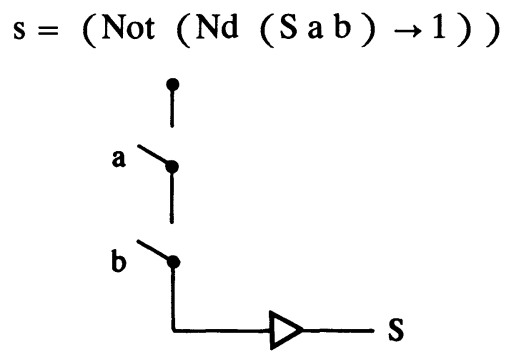

Techno NMOS

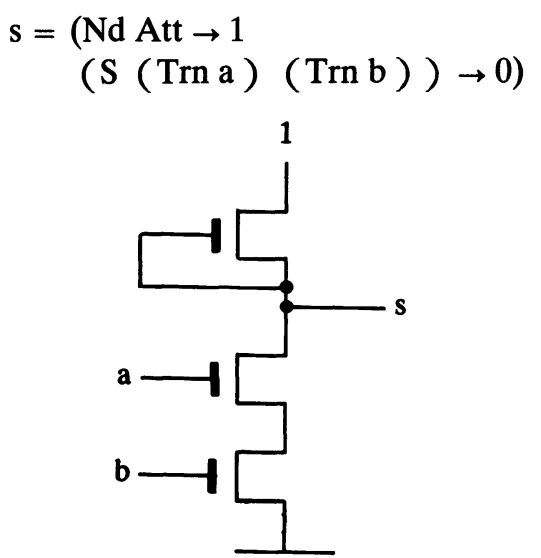

Techno true CMOS

$$
\begin{aligned}
\mathrm{s}=(\operatorname{Nd}(\mathrm{P}(\operatorname{Trp} \mathrm{a}) & (\operatorname{Trp} \mathrm{b})) \\
(\mathrm{S}(\operatorname{Trn} \mathrm{a}) & (\operatorname{Trn} \mathrm{b}))
\end{aligned}
$$

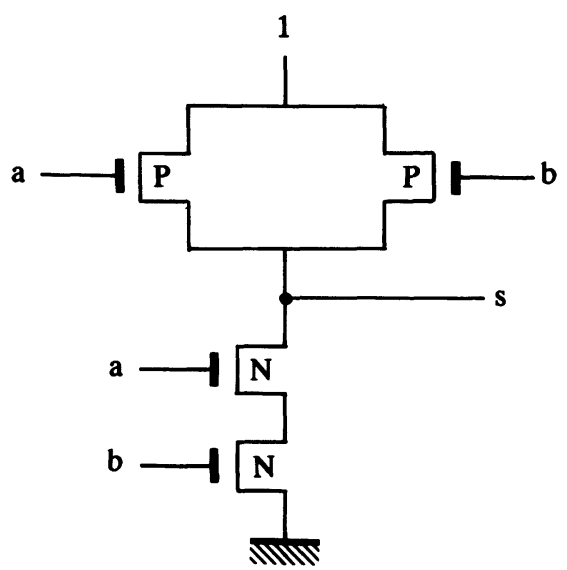


Techno precharged CMOS

$$
\begin{aligned}
& \mathrm{s}=(\mathrm{Nd}(\operatorname{Trp} \text { phi }) \rightarrow 1 \\
&(\mathrm{~S}(\operatorname{Trn} \mathrm{phi})(\operatorname{Trn} \mathrm{a})(\operatorname{Trn} \mathrm{b})) \rightarrow 0)
\end{aligned}
$$

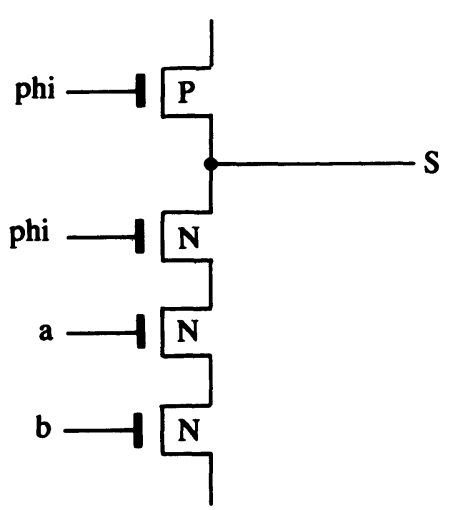

3.5 A USEFUL COMPLEMENT TO SYNTHESIS TOOLS : AUTOMATIC SCHEMATIC. - The previously described formular descriptions are rapidly difficult to read and understand; therefore a graphical representation of them is soon necessary to appreciate quickly the result of synthesis tools. The different algorithms which are beeing used for automatic schematic generation would be the subject of a full paper. Therefore we shall only mention the general principles which have been used to develop this schematic drawing.
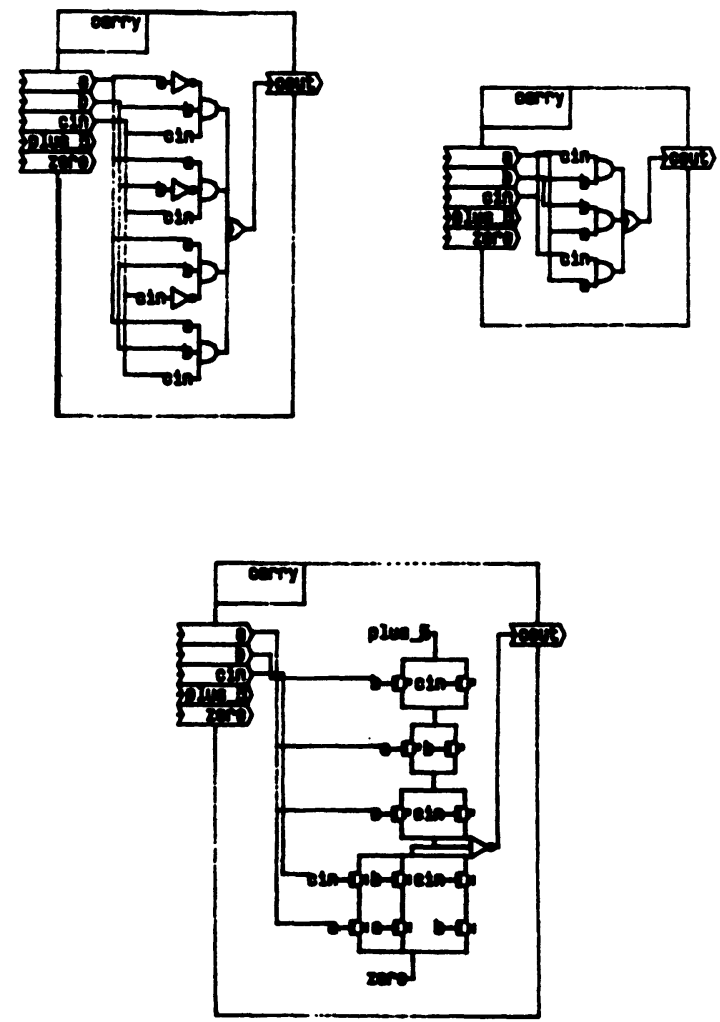

The first objective is that this schematic must be easily readable by designers. The first basic principle which has been chosen is that signals are generally going from the left to the right.

Outputs are generally on the right side of a box inputs are on the left bidirectional signals are generally drawn downwards. This program invokes as one can think placement and routing algorithms ; very roughly bloc placement is done on a flexible prid taking into account the area of each bloc. Routing is done on horizontal and vertical channels between the rows and lines of blocs. The width of each of these channels is proportional to the number of signals in them.

The reader will find in the following pages some examples of such automatic schematics which have been drawn by the system.

\section{Logical extraction.}

The goal of logical extraction is to extract from a transistor network the logical functionality which is achieved by this network.

As for synthesis tools logical extraction is done through sets of rewriting rules.

We shall describe some of these extraction rules. First we have admittances reduction rules. If two admittances $Y_{1}$ and $Y_{2}$ are connected to the same source ( 01 or other) they can be reduced to a single admittance composed of the two assembled in parallel. Let $Y$ be an admittance. We shall also note $Y$ the Boolean function describing $\mathrm{Y}$ in terms of Boolean operators acting on variables which are the signals that activate the transistors of $\mathrm{Y}$. This function is equal to 1 when the admittance is on. For example if $\mathrm{Y}=$ ( $S(\operatorname{Trn} a)(\operatorname{Trn} b))$ the corresponding function is $\mathrm{Y}=($ And $\mathrm{a} \mathrm{b})$. We can then introduce a new reduction rule; any admittance connected to a source different from 0 or $1 S_{1}$ is equivalent to two admittances :

$$
\begin{aligned}
& Y \cdot S_{1} \text { connected to } 1 \\
& Y \cdot / S_{1} \text { connected to } 0
\end{aligned}
$$<smiles>[Y19]C(C)=CC</smiles>

Systematic application of these rules reduces any node to a connexion of two admittances one connected to 1 the other to 0 . Logical extraction can then be reduced 
to the extraction of the canonical node $s=$ $\left(\mathrm{Nd} \mathrm{Y}_{1} \rightarrow 1 \mathrm{Y}_{2} \rightarrow 0\right)$.

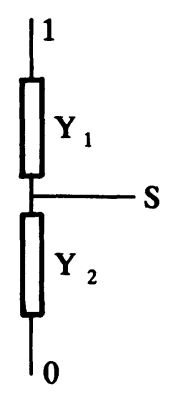

Some necessary conditions have to be satisfied for a node to have a Boolean functionality. First there must not be any short between power and ground. That means that $Y_{1}$ and $Y_{2}$ cannot be both on. In other words the Boolean reduction of $Y_{1} \cdot Y_{2}=$ (And $Y_{1} Y_{2}$ ) must be equal to 0 . The Boolean reducer is used to check this condition.

Except for precharged CMOS one of the two admittances has to be on (equal to 1) which can be checked proving that the Boolean reduction of $/ Y_{1} \cdot / Y_{2}$ is 0 .

When these conditions are satisfied the node has value 1 when $Y_{1}$ is on and $Y_{2}$ is off. The Boolean functionality of the node is therefore

$$
\mathrm{Y}_{1} \cdot / \mathrm{Y}_{2}=\left(\text { And } \mathrm{Y}_{1}\left(\operatorname{Not} \mathrm{Y}_{2}\right)\right)=\mathrm{Y}_{1} \text {. }
$$

Recursive application of all these rules to a transistor network will extract the functionality of each of its output. More subtile rules have to be introduced for precharged CMOS. The same signal phi has to be found in both admittances activating two transistors (One $\mathrm{P}$ and one $\mathrm{N}$ ). Precharge is done for phi $=0$. For phi $=1$ connexion to 1 is off and the logical value is $/ \mathrm{Y}_{2}$.

In conclusion this mechanism not only allows logical extraction but also performs some electrical checking (no shorts no undefined nodes). Similar rules can be developed for logical glitch detection.

\section{Electrical extraction, uniqueness for formulae de- scription.}

When trying to prove the isomorphism of a layout and a formulae description two subproblems arise. From a netlist is it possible to extract a formular description and is this description unique.

\subsection{EXTRACTION OF A FORMULAR DESCRIPTION. -} This extraction is done through two main steps ; first one is admittance construction, second one is formulae construction. The different used algorithm will only be outlined in this paper.

First build a graph whose nodes are the different signals. Two nodes are connected by one edge if they are connected to the source and drain of one same transistor.

Admittance construction :

(i) try to build the longest chains of transistors or admittances assembled in series. When you cannot find any more go to step (ii) ;

(ii) try to assemble chains in parallels. If you cannot find any stop. If you found some but cannot find any more go to (i).

These two steps can be seen and formalized on the previous graph as a graph reduction process.

Formulae construction.

Start from each of the outputs and for each of them create a node formula with arguments which are the different previously built admittances connected to power ground or other nodes. Apply recursively the same procedure to nodes which are neither inputs or power or ground.

5.2 UNIQUENESS OF THE DESCRIPTION. - Since $P$ and $S$ operators are commutative operators the description formulae cannot be unique but it can be proven that the obtained formulae are unique modulo this commutativity. The equivalence of two formulae can therefore be proven by a simplified unification algorithm in which no substitution is needed.

\section{Unification algorithm and rule filtering.}

The commutative unifier has a central role in the system therefore its performances are absolutely critical. A great effort has been devoted to inference engine performance. The total number of rules in the system is several hundreds the Boolean disjunctive reducer has approximatively a hundred rules alone. It has then become necessary to introduce a filtering mechanism on the rules which prevent from useless unification attempts. An example of a simplified distributivity rule will clarify this filtering concept. Let us consider the rule

$$
(\text { And }(\text { Or } a b) c) \rightarrow(\text { Or }(\text { And } a c)(\text { And b c }))
$$

In which $c$ is of type symbol which means that it can only be substituted by a symbol.

For such a rule to be applicable to one expression $f$ the following conditions have to be satisfied:

- f must be an (And ...) expression

- among the operands of this And there must exist a subexpress of type (Or ...)

- among the same list of operands there must exist one symbol.

This type of properties of a list of operands can be recognized in one linear search over the expression and keeps valid for all candidate rules which can then be filtered. 
Nearly ten of such filtering properties have been introduced and they have reduced the number of unification attempts by a ratio of ten to a hundred. The performances of the symbolic calculus tools have been improved by the same ratio. The reader will note that these properties of the operand list can be used in any formal system whatever its operators are.

\section{Conclusion.}

The formalism described above has allowed to develop a full logical and electrical Macsyma like expert system which combines efficiency and safety of design. Some extensions of the system are still to be completed such as automatic sizing of transistors, symbolic simulators, conflict detection and others. The whole system has been written in Lelisp (by Inria) and its object oriented extension Ceyx which has appeared to be the best existing language for this type of development. One possible interesting future extension would be to introduce topological concepts in view of building some general purpose silicon compilers. One very important feature of such a rule based system is that it is easily extensible; some new synthesis tools can be added to the system it is can be adapted to different kinds of digital technologies. One question is still unanswered : it is possible to build a similar formalism for analog circuitry? 